\title{
Neuroimaging in Patients with Abnormal Blood Glucose Levels
}

\author{
G. Bathla, B. Policeni, and A. Agarwal
}

\begin{abstract}
SUMMARY: Smooth neuronal functioning requires an uninterrupted supply of energy that is provided by glucose under normal physiologic conditions. Significant variations in plasma glucose levels, be it hypoglycemia or hyperglycemia, can present with myriad clinical manifestations and may mimic stroke. At times, the diagnosis is either not apparent or not clinically suspected. Imaging can suggest the diagnosis in unsuspected cases and can help in the assessment of the extent of neuronal damage in known cases, making it vital for the neuroradiologist to be aware of both common and atypical neuroimaging findings in hypoglycemia and hyperglycemia.
\end{abstract}

ABBREVIATIONS: GABA = gamma aminobutyric acid; $\mathrm{HC}-\mathrm{HB}=$ hemichorea-hemiballismus; NIDDM = non-insulin-dependent diabetes mellitus; NKHG $=$ nonketotic hyperglycemia

$\mathbf{T}$ he brain relies heavily on a continuous supply of glucose for optimal function. Because the neurons have a high metabolic rate and can neither generate nor store significant amounts of glucose, a rapid decline in plasma glucose can jeopardize neuronal homeostasis over a period of minutes. ${ }^{1}$

Relatively stable plasma glucose levels are therefore essential for survival and are maintained through a highly complex network of enzymes, hormones, and signaling mechanisms, with insulin playing a dominant role. ${ }^{1}$ Enterically absorbed glucose is transported by the portal circulation to the liver, which is the primary organ involved in glucose homeostasis. Here, excess glucose is converted to glycogen and stored for subsequent use. A drop in plasma glucose level triggers a fall in plasma insulin levels, increased glucagon secretion, glycogenolysis, and gluconeogenesis (primarily in the liver) and helps to restore plasma glucose levels whenever needed. ${ }^{1}$

Uptake of glucose in most cells (except liver and brain) is dependent on insulin and is mediated through glucose transporters or occurs through a sodium-glucose co-transport mechanism (small bowel and renal tubules). ${ }^{2}$ Neuronal uptake of glucose is independent of insulin and is mediated through glucose transporter 1. Absorbed glucose is then metabolized to carbon dioxide,

From the Department of Radiology (G.B., B.P.), University of lowa Hospitals and Clinics, lowa City, lowa; and Penn State College of Medicine (A.A.), Milton S. Hershey Medical Center, Hershey, Pennsylvania.

Please address correspondence to Dr. Girish Bathla, Department of Radiology, University of lowa Hospitals and Clinics, 20 Hawkins Dr, lowa City, IA 52242; e-mail: Girish-bathla@uiowa.edu

- Indicates open access to non-subscribers at www.ajnr.org

http://dx.doi.org/10.3174/ajnr.A3486

thereby generating adenosine triphosphate in a process that is heavily oxygen-dependent. Under anaerobic conditions, however, glucose can only be metabolized to produce lactic acid, a process that is not only energy-inefficient but also leads to metabolic acidosis. ${ }^{2}$

Physiologic variations in plasma glucose levels occur during sleep, postprandial states, and as part of circadian rhythm. ${ }^{3} \mathrm{Ab}-$ normal variations in plasma glucose levels may occur in neonates, diabetics, and patients with insulin-secreting tumors, sepsis, Addison disease, and hepatic or renal failure. ${ }^{4-7}$

Significant variations in glucose levels are often symptomatic. Hyperglycemic patients may present with $\mathrm{HC}-\mathrm{HB}$, weakness, hypotonia, pyramidal tract signs, and seizures. ${ }^{8-10}$ Similar findings may occur in hypoglycemic patients, who can additionally present with chorea, ataxia, paresis, aphasia, and coma. ${ }^{8,11-13}$ Neonates with hypoglycemia may show a constellation of symptoms that are usually nonspecific and include poor feeding, lip smacking, eye rolling, and seizures. ${ }^{4,14}$

In emergency settings, it is not uncommon for these symptoms be diagnosed and treated initially as stroke. Imaging in such cases can play a vital role by suggesting the correct diagnosis and ensuring early treatment. This is vital because delayed diagnosis affects morbidity and mortality.

In acute settings, these patients invariably undergo an NCCT of the head initially. It may be occasionally useful but is often nondiagnostic. MR imaging is the study of choice in these cases and is often used to determine diagnosis and prognosis.

As mentioned above, patients with hyperglycemia can present with various movement disorders. However, well-established 
neuroimaging findings are only described in patients with NKHG who have either HC-HB or seizures. At times, patients with ketotic hyperglycemia can also present with movement disorder, though these cases lack any well-described imaging correlate. ${ }^{9}$

Similarly, adults and neonates with significant hypoglycemia can have varying symptoms. However, these are often a consequence of diffuse parenchymal involvement, and findings on imaging are often not correlated with specific symptoms, as seen with hyperglycemia. A notable exception to this is the development of hemiplegia in a subset of adult hypoglycemic patients that correlates with involvement of the contralateral internal capsule. ${ }^{15}$

The following discussion concentrates on hyperglycemic and hypoglycemic conditions that have well-established neuroimaging findings. In hyperglycemic patients, these conditions include HC-HB and seizures. This is followed by a review of imaging findings in neonatal and adult hypoglycemia, in which extensive involvement often precludes correlation with specific symptoms.

\section{Hemichorea-Hemiballismus in Non-Ketotic Hyperglycemia}

HC-HB refers to an involuntary, nonrhythmic, and poorly patterned movement disorder that involves one side of the body. ${ }^{16,17}$ It most commonly results from a vascular insult involving the contralateral basal ganglia. ${ }^{16-18}$ NKHG is the second most common cause for such presentation. ${ }^{16}$ Occasionally, HC-HB may result from neoplastic or infective conditions involving the brain. ${ }^{17,19}$

HC-HB in association with hyperglycemia is well recognized. ${ }^{8}$ It has been predominantly reported in elderly patients with NIDDM and is more common in Asians. ${ }^{17,18,20}$

Patients present with acute onset of $\mathrm{HC}-\mathrm{HB}$, which is often unilateral but may be generalized. ${ }^{8,20}$ The abnormal movements may be continuous or intermittent and disappear during sleep. ${ }^{8,21}$ Laboratory findings confirm poorly controlled diabetes with raised blood glucose and $\mathrm{HbAlC}$ levels and absence of blood and urinary ketones. The serum osmolality is often elevated. ${ }^{19}$ Correction of underlying hyperglycemia is usually curative, leading to rapid cessation of abnormal movements within hours to a few days. ${ }^{8,18}$ Occasionally, however, symptoms may persist for months and require additional suppressant therapy. ${ }^{8}$

The abnormal movements are thought to be secondary to reduced cerebral perfusion, presumably caused by hyperviscosity secondary to hyperglycemia. ${ }^{16,19}$ This is supported by recent observations that raised plasma glucose levels directly correlate with cerebral hypoperfusion. ${ }^{22}$ Ensuing anaerobic metabolism leads to depletion of GABA, which is used as a metabolite to generate energy. ${ }^{19}$ Because GABA is an inhibitory neurotransmitter, its depletion causes increased thalamocortical drive, resulting in HC-HB. ${ }^{16}$

The pathogenesis of the imaging findings in these patients is controversial and is further compounded by variable histopathology results from different studies. ${ }^{8,16}$ Focal hemorrhage and calcification were initially proposed to explain the imaging pattern on CT and MR imaging. However, these are not consistent features on histopathology studies. ${ }^{8}$ The imaging patterns on MR imaging and resolution of findings on follow-up imaging in most

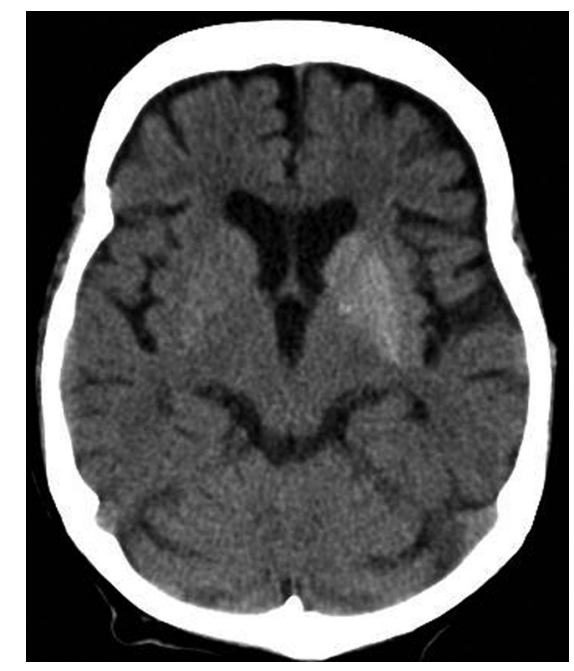

FIG 1. Axial NCCT image reveals asymmetric hyperattenuation involving the lentiform and caudate nuclei bilaterally, more pronounced on the left side.

cases also do not favor hemorrhage or calcification as a common finding. ${ }^{16}$ Some authors have reported the presence of gliotic tissue with abundant gemistocytes (a form of reactive astrocytes), which may account for the T1 shortening on MR imaging. These usually appear during acute injury and can persist for years. ${ }^{16,21}$ These astrocytes can accumulate manganese after periods of brief ischemia, which presumably results from a combination of underlying hyperviscosity and chronic vascular disease. ${ }^{8,16,23,24}$ This is supported by similar signal changes in animals and humans after episodes of transient, nonlethal ischemia, unrelated to hyperglycemia. ${ }^{25}$

The gemistocyte theory does not explain the changes seen on NCCT, leading some authors to speculate that the findings on CT, MR imaging, and associated HC-HB may reflect different pathologic mechanisms running in parallel. ${ }^{21}$ It is possible that the pathogenesis of imaging findings in NKHG is more complex, and transient ischemia may not be the only factor. Hyperglycemia is known to cause endothelial dysfunction and increased oxidative stress in ischemic brain tissue. ${ }^{26}$ These may also contribute to changes seen on imaging, though their precise role is yet to be established.

NCCT often reveals abnormal hyperattenuation, predominantly involving the putamen contralateral to the symptomatic side (Fig 1). The caudate and globus pallidus may be involved. ${ }^{19}$ Patients with ballism may show bilateral abnormalities that can be asymmetric. There is no surrounding edema or mass effect. ${ }^{8}$ Follow-up imaging may show resolution of changes after correction of hyperglycemia. ${ }^{19}$ Occasionally, the initial scan may be normal. ${ }^{8,19}$

On NCCT, the findings are fairly characteristic in the appropriate context, though a similar appearance may occur with hemorrhage or asymmetric calcifications. ${ }^{19}$ Patients receiving intraarterial reperfusion therapy for stroke can also show transient hyperattenuation of the lentiform and insular cortex secondary to interstitial contrast extravasation. ${ }^{27}$ Finally, because the hyperdensities in diabetic patients with HC-HB may resolve slowly or persist for years, ${ }^{28}$ these may reflect previous hyperglycemia-induced injury. 

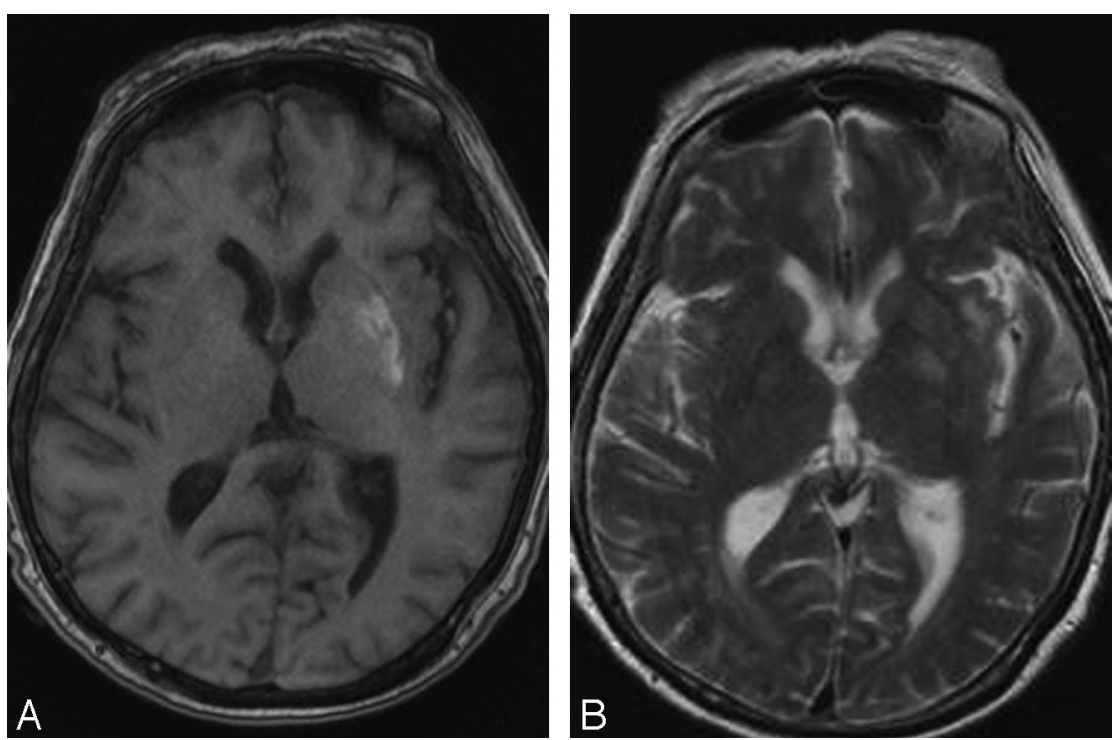

FIG 2. Axial noncontrast TIWI in a patient with HC-HB reveals T7 shortening in the left lentiform. $\mathrm{T} 2 \mathrm{Wl}$ at the same level (B) reveals mild $\mathrm{T} 2$ prolongation.
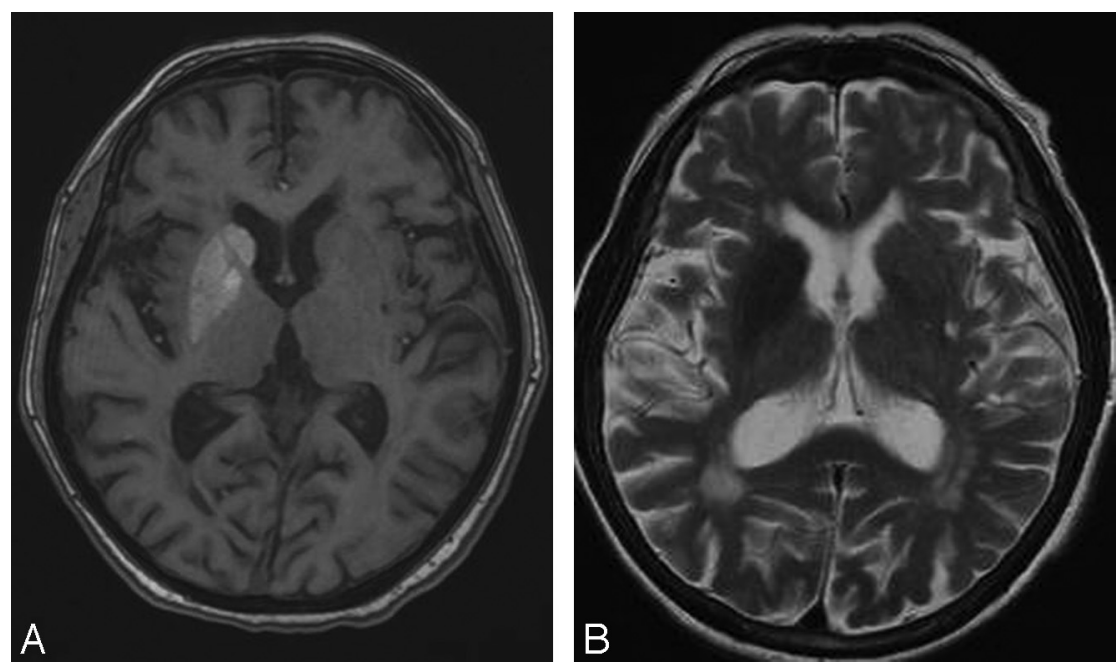

FIG 3. Axial TTWI (A) and T2WI (B) at the level of basal ganglia reveal $\mathrm{T} 1$ and $\mathrm{T} 2$ shortening involving the right corpus striatum.

MR imaging may also reveal abnormal putaminal signal with or without involvement of the caudate and globus pallidus. ${ }^{8}$ As with CT, changes may be bilateral. ${ }^{19}$ Sporadic cases with isolated involvement of subthalamic nuclei have been reported. ${ }^{17}$ The involved regions show $\mathrm{T} 1$ shortening. ${ }^{29}$ The signal changes on T2WI are variable, and the lesions may manifest either T2 shortening or prolongation (Figs 2 and 3). ${ }^{17,19}$ Restricted diffusion and signal loss on gradient-echo images have been reported occasionally. ${ }^{19,30,31}$ Some authors have reported abnormal mineral deposition in the affected regions on susceptibility-weighted MR imaging. ${ }^{16}$ Additionally, dilated vessels over the affected side have been reported and are presumed to reflect underlying ischemia. ${ }^{29}$

Data are limited on $1 \mathrm{H}-\mathrm{MR}$ spectroscopy findings, with the reported cases showing reduction of NAA/Cr ratio, elevation of Cho/Cr ratio, and presence of a lactate peak. ${ }^{8,23}$ Similarly, sporadic case reports of SPECT show hypoperfusion within the basal ganglia, corresponding to the symptomatic side. ${ }^{19}$
The imaging findings are usually reversible, though they lag behind clinical improvement. Occasionally, the abnormalities persist and have been reported up to 6 years after the initial presentation. ${ }^{28}$

Besides HC-HB, T1 shortening of the basal ganglia may occur with chronic liver disease, manganese deposition, neurofibromatosis type 1 , and calcifications. ${ }^{32}$ However, a quick review of the patient chart usually excludes most of these considerations.

\section{Seizures in Non-Ketotic Hyperglycemia}

Seizures are relatively common in patients with NKHG. ${ }^{33}$ These are often focal, with the most common subtype being focal motor seizure with or without secondary generalization. ${ }^{34-36}$ Less frequently, occipital lobe seizures or epilepsia partialis continua may also occur. ${ }^{10,33,35}$ MR imaging in these patients may show areas of transient T2 prolongation, which are thought to be secondary to the ictus and may be seen with seizures unrelated to hyperglycemia. However, some authors have reported subcortical areas of T2 shortening in a subset of patients with NKHG, which often correlate with localization of ictal focus on EEG studies and regress after normalization of hyperglycemia. ${ }^{37}$ Therefore, these are more likely to be related to the underlying hyperglycemic state.

As with $\mathrm{HC}-\mathrm{HB}$, these patients also show evidence of poorly controlled diabetes and underlying hyperosmolality. ${ }^{10}$ The HbA1C is often elevated, attesting to long-term hyperglycemia. ${ }^{33,34}$ In some cases, the seizures may be the first presentation and uncover underlying diabetes. ${ }^{34,35}$

Similar to $\mathrm{HC}-\mathrm{HB}$, correction of underlying hyperglycemia is curative. ${ }^{10,33}$ Anti-epileptic drugs are often not required because the seizures respond promptly to correction of hyperglycemia. ${ }^{35}$ Occasional cases may require short-term phenobarbital or valproic acid. ${ }^{35}$ Phenytoin is avoided because it worsens glycemic control and can aggravate seizures. ${ }^{34}$

A predilection for occipital lobe involvement exists. ${ }^{34,36} \mathrm{~Pa}$ tients often present with visual symptoms such as blurring, field defects, and hallucinations. The parietal, temporal, and perirolandic cortex also may be involved. ${ }^{33,37}$

It is unclear how hyperglycemia precipitates seizures. Elevated blood glucose levels have a pro-convulsant effect and reduce seizure threshold. ${ }^{34-36}$ As pointed out earlier, a hyperglycemic state also induces cellular anaerobic metabolism and depletes GABA, which is an inhibitory neurotransmitter. ${ }^{35}$ The presence of con- 
current ketoacidosis is thought to offer some protection against seizures because ketone bodies can serve as substrate for GABA generation. $^{36}$

The reason for subcortical T2 shortening on imaging is also unclear. Postulated mechanisms include mineral deposition and ischemia. ${ }^{33,34,38}$ A hypointense signal on the gradient-echo image would support free radical accumulation. ${ }^{33}$

NCCT is often negative, though rare cases with both HC-HB and seizures may show basal ganglia hyperattenuation. ${ }^{38,39} \mathrm{On}$ MR imaging, the involved regions are usually isointense on T1WI but may show focal T2 prolongation in the cortex and T2 shortening in the underlying WM. ${ }^{33-35}$ Similar hypointense subcortical signal is also noted on gradient-echo images. ${ }^{33}$ Associated cortical swelling is often appreciable on T2WI/ FLAIR images. Transiently reduced $\mathrm{ADC}$ values and focal cortical or leptomeningeal enhancement may occur. ${ }^{33,35}$ These findings often completely resolve over a period of months, though some cases show focal gliosis. ${ }^{33}$

Sporadic reports of ${ }^{1} \mathrm{H}-\mathrm{MR}$ spectroscopy have described reduced NAA in the involved regions. ${ }^{38}$ There are few case reports of ictal hyperperfusion in the involved regions on technetium Tc99m hexamethylpropyleneamine oxime SPECT imaging, in contrast to HC-HB in a similar subgroup of patients, which shows hypoperfusion. ${ }^{19,34,38}$

Besides NKHG, subcortical T2 shortening may also be seen in viral encephalitis, meningitis, hypoxic insult, and Moyamoya disease. ${ }^{33}$ Overlying leptomeningeal enhancement, if present, would add metastasis, sarcoidosis, and lymphoma to the list of differential considerations. However, a known hyperglycemic state and supportive EEG findings may help to clinch the diagnosis.

\section{Neonatal Hypoglycemia}

Transient hypoglycemia is relatively common in neonates as they transition from intrauterine to extrauterine environment. ${ }^{5}$ The incidence of neonatal hypoglycemia is not clearly defined because there is no absolute consensus on the exact blood glucose value to define hypoglycemia. ${ }^{4}$ The plasma glucose level used to define hypoglycemia varies between $25-46 \mathrm{mg} / \mathrm{dL}$ in different studies, though most researchers agree that a level $<36 \mathrm{mg} / \mathrm{dL}$ within first 2-3 hours after birth requires intervention. ${ }^{14,40,41}$

Neuronal damage secondary to hypoglycemia is uncommon but may occur. ${ }^{4,5}$ These children with this damage often present with nonspecific symptoms such as seizures, lip-smacking, poor feeding, hypotonia, cyanosis, and vomiting. ${ }^{4,5,14,41}$

Neonates often have development of hypoglycemia within hours to days of birth. Alkalay et al, ${ }^{14}$ in their review of 23 patients, found that the mean age at presentation was 30 hours and varied between 1-72 hours. Because there is a broad list of differentials for seizures in this age group, these children often undergo a battery of tests to exclude metabolic, congenital, and infective causes before imaging is considered. Transcranial sonography is usually the initial imaging study and is performed to exclude hypoxic or hemorrhagic insults. However, it has low sensitivity for detecting hypoglycemic brain injury. ${ }^{14}$ Because the clinical diagnosis of hypoglycemia can be made with a simple strip test, imaging with CT/MR is often performed to assess the extent of injury and prognosticate final recovery rather than to confirm the diagnosis.

Neuroimaging findings in neonatal hypoglycemia were first reported by Spar et $\mathrm{al}^{42}$ in 1994 . Since then, similar findings have been described in small patient cohorts by various authors. ${ }^{4,14,43}$ A predilection for occipital and parietal lobe involvement exists, the exact reason for which is unclear. ${ }^{4,5,14}$ Some authors have attributed it to relatively higher glucose utilization in this region from intensive axonal growth. ${ }^{14}$ Others attribute the changes to a variable distribution of excitatory neurotransmitters in different parts of brain. ${ }^{4,41}$ The remaining cerebral hemispheres, corticospinal tracts, corpus callosum, and deep gray nuclei also may be affected. ${ }^{4,5,14,40}$ Posterior fossa involvement is rare. ${ }^{5}$ The pattern of brain injury is independent of underlying etiology, essentially implicating hypoglycemia as the culprit. ${ }^{4}$

On CT, the involved regions appear hypoattenuated in the acute phase and are often bilateral. In fact, bilateral occipital involvement is considered by some to be specific for hypoglycemiainduced injury. The gray-white interface may be involved. ${ }^{14,41,43}$ Lesions are often bilateral. ${ }^{33,35}$ Hemorrhage has not been reported on CT studies. Follow-up imaging usually shows volume loss. ${ }^{43}$

On MR imaging, variable signal is noted on most sequences. The involved cortex is usually isointense on T1WI but may show focal T1 shortening. Similarly, the cortex usually shows T2 prolongation, but focal T2 shortening may occur. ${ }^{4,14}$ Underlying WM involvement manifests as areas of T1 and T2 prolongation. ${ }^{4}$ Follow-up imaging often shows parenchymal atrophy. Cystic encephalomalacia may occur. ${ }^{4}$ Some patients may have transient abnormalities that resolve on follow-up. ${ }^{14}$ Not surprisingly, these patients have less severe hypoglycemia and present early. ${ }^{14}$

DWI is most sensitive for identification of parieto-occipital injury, especially in the first 6 days after birth (Fig 4). ${ }^{44}$ In the acute phase, there is restricted diffusion with correspondingly reduced ADC values. ${ }^{14}$ Some authors think that the degree of restricted diffusion may correlate with parenchymal damage on follow-up. ${ }^{45}$ Burns et $\mathrm{al}^{5}$ described presence of focal WM hemorrhagic lesions in up to $30 \%$ of their cases. However, these findings have not been reported in other studies. The reason for this discrepancy is unclear.

Hypoglycemia potentiates hypoxia-induced damage, and a combination of the 2 leads to worse outcomes than hypoxia alone. ${ }^{4,46,47}$ These children often present with nonspecific symptoms such as seizures, lip-smacking, poor feeding, hypotonia, cyanosis, and vomiting. ${ }^{4}$

There is scarce literature on perfusion imaging and spectroscopy findings in these patients. Kim et $\mathrm{al}^{45}$ reported reduced NAA with the presence of a lactate peak in their patient, with further reduction in the NAA peak on follow-up 1H-MR spectroscopy on the 16th day.

On imaging, the most important differential consideration is hypoxic encephalopathy, either alone or in combination with hypoglycemia. When a predominantly posterior distribution is seen, the diagnosis of hypoglycemic injury is relatively straightforward. However, with diffuse bilateral involvement, it may be extremely difficult to exclude hypoxia or a combined insult. A review of the patient's Apgar score, plasma glucose, and arterial blood gas levels may provide important clues. 

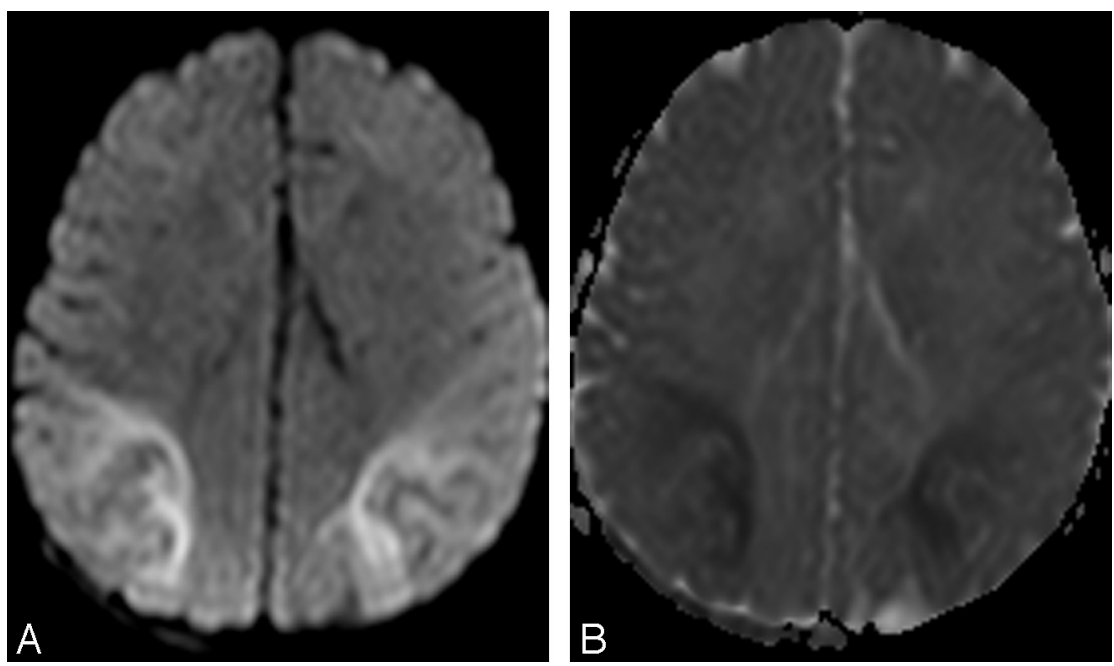

FIG 4. Axial DWI (A) and $A D C$ image $(B)$ in a hypoglycemic neonate reveal restricted diffusion involving predominantly the parietal and occipital regions with corresponding $A D C$ hypointensity.

Prognosis in these children depends on prompt recognition and treatment of hypoglycemia. However, the long-term prognosis can be variable and correlates with WM damage at MR imaging. ${ }^{5}$ The overall outcome may be complicated by occipital lobe epilepsy, visual disturbances, mental retardation, cerebral palsy, and microcephaly. ${ }^{5,14,40}$

\section{Adult Hypoglycemia}

According to the American Diabetes Association, ${ }^{48}$ hypoglycemia and severe hypoglycemia are defined as plasma glucose levels $<70$ $\mathrm{mg} / \mathrm{dL}(3.9 \mathrm{mmol} / \mathrm{L})$ and $40 \mathrm{mg} / \mathrm{dL}(2.2 \mathrm{mmol} / \mathrm{L})$, respectively. Hypoglycemia is more common in diabetic patients and can occur as a complication of therapy with insulin or long-acting sulfonylurea drugs. Symptomatic hypoglycemia is believed to affect $2 \%$ of diabetic patients annually and is associated with up to $4 \%$ of deaths in patients with type 1 diabetes. ${ }^{6,11,49}$ Other causes include exogenous administration of insulin, which may be suicidal or accidental, insulin-secreting tumors, sepsis, Addison disease, and hepatic or renal failure. ${ }^{6,7,11}$

Hypoglycemic symptoms can be divided into autonomic, which include sweating, trembling, palpitations, and anxiety and neuroglycopenic, which include weakness, confusion, personality changes, seizures, and transient memory loss. ${ }^{6,7,15,50,51}$ Severe hypoglycemia may present with altered mental state or coma. ${ }^{6,52-54}$ Occasionally, patients may develop hemiparesis or quadriparesis and mimic a stroke. ${ }^{12,51,53}$ In general, autonomic symptoms usually develop before neuroglycopenic symptoms. However, some diabetic patients may develop "hypoglycemic unawareness," and autonomic symptoms may not develop or may remain unrecognized. ${ }^{11}$

Energy failure leading to loss of cellular hemostasis is believed to be a key factor in hypoglycemia-induced brain damage. ${ }^{6,52-54}$ Many authors have also pointed out the role of aspartate-induced injury in hypoglycemia, in contrast to hypoxia, in which the neuronal damage is secondary to glutamate. ${ }^{15,52,55,56}$ Absence of glucose results in accumulation of oxaloacetate, which in turn leads to generation of excess aspartate. Aspartate is a known neurotoxin and has been shown to cause preferential neuronal necrosis in the cerebral cortex, neostriatum, and hippocampus. ${ }^{6,11,15,50}$

Hypoglycemia was initially reported to predominantly involve the cortex, neostriatum, and hippocampus. ${ }^{6,11,12,54,57}$ However, many authors have also reported predominant WM involvement, mainly affecting the centrum semiovale, corona radiata, internal capsule, an splenium of the corpus callosum. ${ }^{711,12,53,54,56}$ In fact, involvement of the WM is now thought to be earlier and more common than gray matter involvement. ${ }^{52,53}$ Some cases may show diffuse gray matter and WM involvement. ${ }^{6,7,53,54}$ The thalamus, brain stem, and cerebellum are invariably spared, and this may help to differentiate hypoglycemia from hypoxic injury, which often involves the thalamus. ${ }^{6,52,55,56}$

Because these patients often present in an altered mental state, underlying hypoglycemia may not be apparent. NCCT in these patients is often unremarkable. ${ }^{15,49,50}$ Some cases may show nonspecific WM hypodensities or diffuse cerebral edema in the acute stage and parenchymal volume loss on follow-up. ${ }^{15,49}$

On MR imaging, the earliest changes are seen on DWI sequences. ${ }^{52-54}$ The involved regions show restricted diffusion with corresponding ADC hypointensity. ${ }^{6,7,12,15,53}$ The extent of these abnormalities depends on severity and duration of hypoglycemia. ${ }^{6,49,52,57}$ Changes are usually bilateral, though bilaterally asymmetric, or, rarely, unilateral lesions, may occur. ${ }^{11,52,57}$ Reduction in ADC values has been shown to follow establishment of cerebral isoelectricity, a process that may be asynchronous. This may explain asymmetric lesions in a given patient. ${ }^{11,53,58}$

Experimental studies on rats have shown that reduction in ADC values follows cerebral isoelectricity and may quickly normalize after glucose infusion. ${ }^{51}$ It has also been shown that hypoglycemia-induced neuronal damage does not occur until the EEG becomes iso-electric and is independent of blood glucose level. ${ }^{52}$ Establishment of cerebral isoelectricity may be asynchronous, and this may explain asymmetric lesions in a given patient. ${ }^{9,46}$

Contrary to the above observations, Schmidt et al ${ }^{11}$ recently showed that short-term severe hypoglycemia by itself may not show any visible diffusion abnormalities. Although they reported findings in a small number of patients $(n=10)$, none of whom lost consciousness, the findings may lead one to speculate that individual susceptibility and associated comorbidities may also influence imaging findings and clinical outcomes.

Patients with hypoglycemia also may show $\mathrm{T} 2$ prolongation in both cortex and WM. ${ }^{7}$ These regions are usually isointense on T1WI. ${ }^{49}$ However, Fujioka et $\mathrm{al}^{55}$ showed persistence of both T1 and $\mathrm{T} 2$ shortening in 4 patients who entered a persistent vegetative state. Hemorrhagic lesions have not been reported. ${ }^{6,50,55}$ Post-contrast enhancement is usually absent but may occur. ${ }^{6,49,50}$

Sporadic reports on perfusion studies show either no significant change or slightly increased perfusion in affected re-

AJNR Am J Neuroradiol 35:833-40 May 2014 www.ajnr.org 


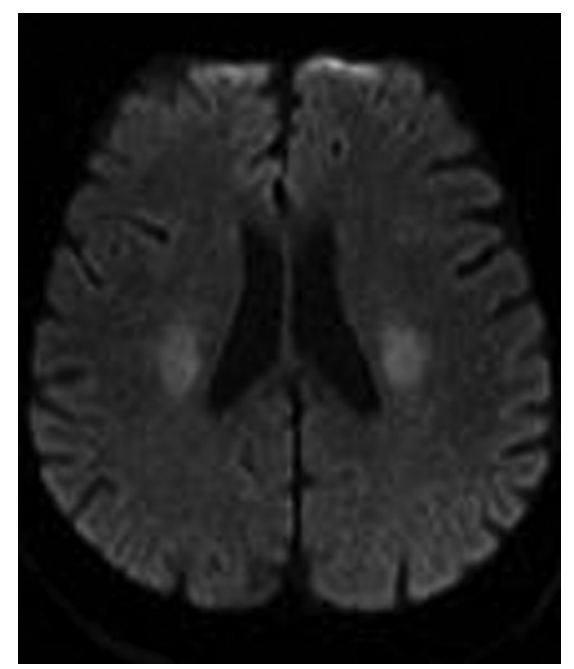

FIG 5. Axial DWI in a hypoglycemic patient reveals restricted diffusion involving the corona radiata bilaterally. The splenium was spared (not shown).

gions. ${ }^{54,57,59}$ Similarly, only sporadic reports about ${ }^{1} \mathrm{H}-\mathrm{MR}$ spectroscopy exist and show mildly reduced NAA and preservation of choline and creatine. ${ }^{57}$ No lactate peak is seen, probably because of the absence of glucose. ${ }^{55,60}$ This may help to distinguish hypoxic from hypoglycemic insults.

On the basis of the topographic distribution of the signal abnormalities, 3 imaging patterns have been described. ${ }^{52}$ These include 1) predominant gray matter involvement affecting the cortex, neostriatum, and hippocampi; 2) predominant WM involvement affecting the periventricular WM, internal capsule, and splenium of corpus callosum (Fig 5); and 3) mixed pattern, involving both the gray matter and WM (Fig 6).

The division of patients on the basis of these imaging patterns is of unclear significance. ${ }^{52,53}$ Patients with focal involvement of the internal capsule, corona radiata, or splenium usually have a good prognosis. ${ }^{6,7,53,54}$ These lesions usually resolve promptly after restoration of blood glucose, though they tend to follow clinical symptom resolution. ${ }^{6,7,12,15,54}$ Atay et $\mathrm{al}^{56}$ reported near total
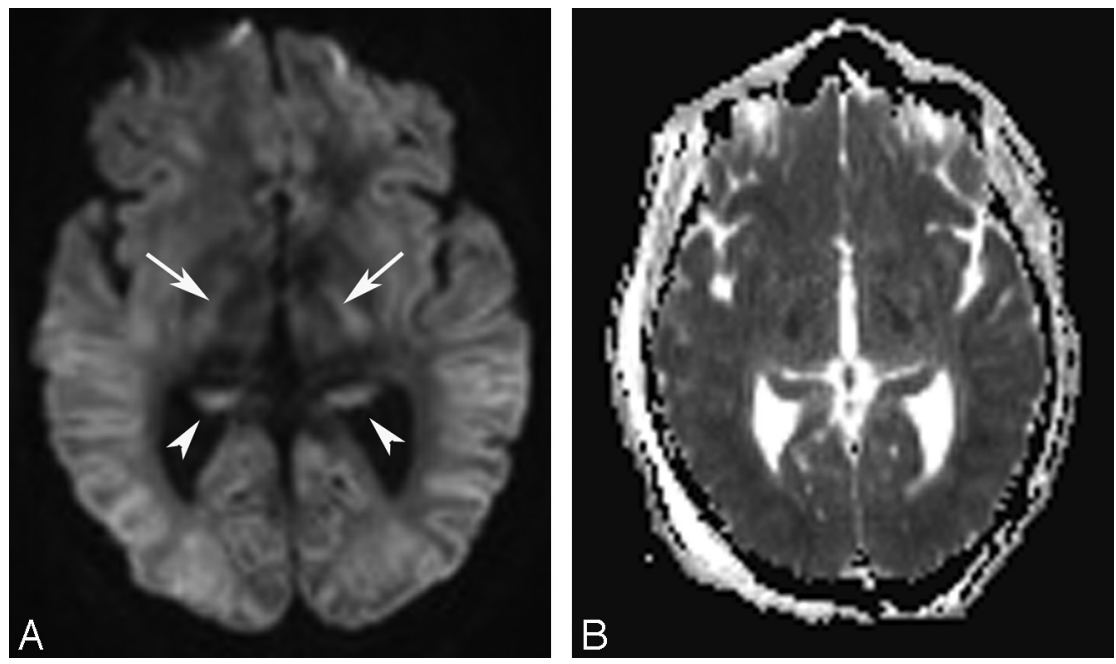

FIG 6. Axial DWI $(A)$ and $A D C$ image $(B)$ in another hypoglycemic patient show restricted diffusion involving the cortex, hippocampi (arrowheads in A), and internal capsules (arrows in A) bilaterally with correspondingly reduced ADC values. resolution of DW abnormalities in their patient as early as 2 hours after blood glucose levels were restored.

Patients with extensive WM involvement show variable response. ${ }^{52}$ The prognosis in these cases varies between complete recovery and persistent vegetative state. ${ }^{53}$ Clinical improvement, if it occurs, is usually delayed by weeks. ${ }^{6,17}$ Involvement of the neostriatum and diffuse cortical lesions often portend dismal outcome. ${ }^{7,49,50,52,57}$ Failure of lesions to regress on follow-up imaging is also associated with poor prognosis. ${ }^{6}$

There is no satisfactory explanation as to why a subgroup of patients with hypoglycemia has hemiparesis and a good prognosis, whereas others show diffuse WM lesions and a variable prognosis, and still others have diffuse gray matter involvement and an invariably poor prognosis. Some authors believe that these probably represent a spectrum of injury and that focal WM changes usually result from transient hypoglycemia. ${ }^{15,52}$ However, some other authors believe that they may be attributable to different pathophysiologies. ${ }^{7,12,52}$ Regardless of the cause, imaging subgroups in hypoglycemia do exist, and DWI probably has some prognostic value.

Patients with extensive cortical and WM involvement often present a diagnostic challenge. Similar changes can also occur with hypoxia, hyperammonemia, encephalitis, after seizures, or may be drug-induced. ${ }^{6,50}$ Splenial lesions have been reported in seizures, anti-epileptic drug withdrawal or toxicity, alcohol abuse, encephalitis, and electrolyte derangements. ${ }^{30,61}$ The clinical context, along with a review of biochemical findings, should help narrow diagnostic considerations. Sparing of the thalamus, brain stem, and cerebellum is a useful clue. In select cases, $1 \mathrm{H}-\mathrm{MR}$ spectroscopy may serve as a problem-solving technique.

\section{CONCLUSIONS}

Abnormal variations in plasma glucose levels are not uncommon and can show myriad imaging findings. The presentation is often nonspecific, and objective findings of $\mathrm{HC}-\mathrm{HB}$, memory loss, hemiparesis, and coma often clinically mimic stroke. A timely and accurate diagnosis would expedite correct treatment and limit neuronal injury in the early stage, when changes are potentially reversible.

\section{REFERENCES}

1. Cryer PE, Davis SN. Hypoglycemia. In: Longo DL, Fauci AS, Kasper DL, et al, eds. Harrison's Principles of Internal Medicine. 18th ed. New York: McGraw-Hill Co; 2012:3003-09

2. Hall JE. Guyton and Hall Textbook of Medical Physiology. Philadelphia: Saunders Elsevier; 2011:809-18

3. Van Cauter E, Polonsky KS, Scheen AJ. Roles of circadian rhythmicity and sleep in human glucose regulation. Endocr Rev 1997;18:716-38

4. Barkovich AJ, Ali FA, Rowley HA, et al. Imaging patterns of neonatal hypoglycemia. AJNR Am J Neuroradiol 1998; 19:523-28 
5. Burns CM, Rutherford MA, Boardman JP, et al. Patterns of cerebral injury and neurodevelopmental outcomes after symptomatic neonatal hypoglycemia. Pediatrics 2008;122:65-74

6. Kang EG, Jeon SJ, Choi SS, et al. Diffusion MR imaging of hypoglycemic encephalopathy. AJNR Am J Neuroradiol 2010;31:559-64

7. Lo L, Tan AC, Umapathi T, et al. Diffusion-weighted MR imaging in early diagnosis and prognosis of hypoglycemia. AJNR Am J Neuroradiol 2006;27:1222-24

8. Jagota P, Bhidayasiri R, Lang AE. Movement disorders in patients with diabetes mellitus. J Neurol Sci 2012;314:5-11

9. Bandyopadhyay SK, Dutta A. Hemifacial spasm complicating diabetic ketoacidosis. J Assoc Physicians India 2005;53:649-50

10. Sabitha KM, Girija AS, Vargese KS. Seizures in hyperglycemic patients. J Assoc Physicians India 2001;49:723-26

11. Schmidt P, Böttcher J, Ragoschke-Schumm A, et al. Diffusionweighted imaging in hyperacute cerebral hypoglycemia. AJNR Am J Neuroradiol 2011;32:1321-27

12. Terakawa $Y$, Tsuyuguchi N, Nunomura K, et al. Reversible diffusionweighted imaging changes in the splenium of the corpus callosum and internal capsule associated with hypoglycemia: case report. Neurol Med Chir (Tokyo) 2007;47:486-88

13. Kossoff EH, Ichord RN, Bergin AM. Recurrent hypoglycemic hemiparesis and aphasia in an adolescent patient. Pediatric Neurol 2001;24:385-86

14. Alkalay AL, Flores-Sarnat L, Sarnat HB, et al. Brain imaging findings in neonatal hypoglycemia: case report and review of 23 cases. Clin Pediatr (Phila) 2005;44:783-90

15. Albayram S, Ozer H, Gokdemir S, et al. Reversible reduction of apparent diffusion coefficient values in bilateral internal capsules in transient hypoglycemia-induced hemiparesis. AJNR Am J Neuroradiol 2006;27:1760-62

16. Cherian A, Thomas B, Baheti NN, et al. Concepts and controversies in nonketotic hyperglycemia-induced hemichorea: further evidence from susceptibility-weighted MR imaging. J Magn Reson Imaging 2009;29:699-703

17. Kim H-J, Moon WJ, Oh J, et al. Subthalamic lesion on MR imaging in a patient with nonketotic hyperglycemia-induced hemiballism. AJNR Am J Neuroradiol 2008;29:526-27

18. Shalini B, Salmah W, Tharakan J. Diabetic non-ketotic hyperglycemia and the hemichorea-hemiballism syndrome: a report of four cases. Neurology Asia 2010;15:89-91

19. Lai PH, Tien RD, Chang MH, et al. Chorea-ballismus with nonketotic hyperglycemia in primary diabetes mellitus. AJNR Am J Neuroradiol 1996;17:1057-64

20. Wintermark M, Fischbein NJ, Mukherjee P, et al. Unilateral putaminal CT, MR, and diffusion abnormalities secondary to nonketotic hyperglycemia in setting of acute neurologic symptoms mimicking stroke. AJNR Am J Neuroradiol 2004;25:975-76

21. Shan DE. Hemichorea-hemiballism associated with hyperintense putamen on T1-weighted MR images: an update and a hypothesis. Acta Neurol Taiwan 2004;13:170-77

22. Cosentino F, Battista R, Scuteri A, et al. Impact of fasting glycemia and regional cerebral perfusion in diabetic subjects: a study with technetium-99m-ethyl cysteinate dimer single photon emission computed tomography. Stroke 2009;40:306-08

23. Lai $\mathrm{PH}$, Chen $\mathrm{PC}$, Chang MH, et al. In vivo proton MR spectroscopy of chorea-ballismus in diabetes mellitus. Neuroradiology 2001; 43:525-31

24. Fujioka M, Taoka T, Matsou Y, et al. Magnetic resonance imaging shows delayed ischemic striatal neurodegeneration. Ann Neurol 2003; $54: 732-47$

25. Baheti NN, Cherian A, Wattamwar PR, et al. Ischemic hyperintensities on T1-weighted magnetic resonance imaging of patients with stroke: new insights from susceptibility weighted imaging. Neurol India 2010;58:90-94

26. Bemeur C, Ste-Marie L, Montgomery J. Increased oxidative stress during hyperglycemic cerebral ischemia. Neurochem Int 2007; 50:890-904
27. Nakano S, Iseda T, Kawano H, et al. Parenchymal hyperdensity on computed tomography after intra-arterial reperfusion therapy for acute middle cerebral artery occlusion: incidence and clinical significance. Stroke 2001;32:2042-48

28. Shan DE, Ho DM, Chang C, et al. Hemichorea-hemiballism: an explanation for MR signal changes. AJNR Am J Neuroradiol 1998;19:863-70

29. Mittal P. Hemichorea-hemiballism syndrome: a look through susceptibility weighted imaging. Ann Indian Acad Neurol 2011;14:124-26

30. Massaro F, Palumbo P, Falcini M, et al, Generalized chorea-ballism in acute non ketotic hyperglycemia: findings from diffusionweighted magnetic resonance imaging. Parkinsonism Relat Disord 2012;18:998-99

31. Chu K, Kang DW, Kim DE, et al. Diffusion-weighted and gradient echo magnetic resonance findings of hemichorea-hemiballismus associated with diabetic hyperglycemia: a hyperviscosity syndrome? Arch Neurol 2002;59:448-52

32. Rovira A, Alonso J, Córdoba J. MR imaging findings in hepatic encephalopathy. AJNR Am J Neuroradiol 2008;29:1612-21

33. Raghavendra S, Ashalatha R, Thomas SV, et al. Focal neuronal loss, reversible subcortical focal $\mathrm{T} 2$ hypointensity in seizures with a nonketotic hyperglycemic hyperosmolar state. Neuroradiology 2007;49:299-305

34. Hung WL, Hsieh PF, Lee YC, et al. Occipital lobe seizures related to marked elevation of hemoglobin A1C: report of two cases. Seizure 2010;19:359-62

35. Lin WS, Kao HW, Sung YF. Reversible magnetic resonance imaging abnormality in a case of diabetic hyperglycemia related epilepsia partialis continua. J Intern Med Taiwan 2011;22:438-43

36. Moien-Afshari F, Téllez-Zenteno JF. Occipital seizures induced by hyperglycemia: a case report and review of literature. Seizure 2009;18:382-85

37. Seo DW, Na DG, Na DL, et al. Subcortical hypointensity in partial status epilepticus associated with nonketotic hyperglycemia. J Neuroimaging 2003;13:259-63

38. Wang CP, Hsieh PF, Chen CC, et al. Hyperglycemia with occipital seizures: images and visual evoked potentials. Epilepsia 2005; $46: 1140-44$

39. Chung SJ, Lee JH, Lee SA, et al. Co-occurrence of seizure and chorea in a patient with nonketotic hyperglycemia. Eur Neurol 2005;54:230-32

40. Tam EWY, Haeusslein BA, Bonifacio SL. Hypoglycemia is associated with increased risk for brain injury and adverse neurodevelopmental outcome in neonates at risk for encephalopathy. $J$ Pediatr 2012;161:88-93

41. Karimzadeh P, Tabarestani S, Ghofrani M. Hypoglycemia-occipital syndrome: a specific neurologic syndrome following neonatal hypoglycemia? J Child Neurol 2010;26:152-59

42. Spar JA, Lewine JD, Orrison WW Jr. Neonatal hypoglycemia: CT and MR findings. AJNR Am J Neuroradiol 1994;15:1477-78

43. Traill Z, Squier M, Anslow P. Brain imaging in neonatal hypoglycaemia. Arch Dis Child Fetal Neonatal Ed 1998;79:F145-F147

44. Tam EWY, Widjaja E, Blaser SI, et al. Occipital lobe injury and cortical visual outcomes after neonatal hypoglycemia. Pediatrics 2008;122:507-12

45. Kim SY, Goo HW, Lim KH, et al. Neonatal hypoglycaemic encephalopathy: diffusion-weighted imaging and proton MR spectroscopy. Pediatr Radiol 2006;36:144-48

46. Basu P, Som S, Choudhuri N, et al. Contribution of the blood glucose level in perinatal asphyxia. Eur J Pediatr 2009;168:833-38

47. Nadeem M, Murray DM, Boylan GB, et al. Early blood glucose profile and neurodevelopmental outcome at two years in neonatal hypoxic-ischaemic encephalopathy. BMC Pediatr 2011;11:10

48. American Diabetes Association. Standards of medical care in diabetes-2012. Diabetes Care 2012;35(Suppl 1):S11-S63

49. Finelli PF. Diffusion-weighted MR in hypoglycemic coma. Neurology 2001;57:933

AJNR Am J Neuroradiol 35:833-40 May 2014 www.ajnr.org 
50. Chan R, Erbay S, Oljeski S, et al. Case report: hypoglycemia and diffusion-weighted imaging. J Comput Assist Tomogr 2003;27:420-23

51. Yoshino T, Meguro S, Soeda Y, et al. A case of hypoglycemic hemiparesis and literature review. Ups J Med Sci 2012;117:347-51

52. Ma JH, Kim YJ, Yoo WJ, et al. MR imaging of hypoglycemic encephalopathy: lesion distribution and prognosis prediction by diffusion-weighted imaging. Neuroradiology 2009;51:641-49

53. Johkura K, Nakae Y, Kudo Y, et al. Early diffusion MR imaging findings and short-term outcome in comatose patients with hypoglycemia. AJNR Am J Neuroradiol 2012;33:904-09

54. Böttcher J, Kunze A, Kurrat C, et al. Localized reversible reduction of apparent diffusion coefficient in transient hypoglycemia-induced hemiparesis. Stroke 2005;36:e20-e22

55. Fujioka M, Okuchi K, Hiramatsu K, et al. Specific changes in human brain after hypoglycemic injury. Stroke 1997;28:584-87

56. Atay M, Aralasmak A, Sharifov R, et al. Transient cytotoxic edema caused by hypoglycemia: follow-up diffusion weighted imaging features. Emerg Radiol 2012;19:473-75

57. Lim CC, Gan R, Chan CL, et al. Severe hypoglycemia associated with an illegal sexual enhancement product adulterated with glibenclamide: MR imaging findings. Radiology 2009;250: 193-201

58. Hasegawa Y, Formato JE, Latour LL, et al. Severe transient hypoglycemia causes reversible change in the apparent diffusion coefficient of water. Stroke 1996;27:1648-56

59. Cordonnier C, Oppenheim C, Lamy C, et al. Serial diffusion and perfusion-weighted MR in transient hypoglycemia. Neurology 2005;65:175

60. Auer RN, Siesjo BK. Biological differences between ischemia, hypoglycemia, and epilepsy. Ann Neurol 1988;24:699-707

61. Gracia-Monco JC, Cortina IE, Ferreira E, et al. Reversible splenial lesion syndrome (RESLES): what's in a name? J Neuroimaging 2011; 21:e1-e14 